\title{
High fidelity optical modeling for the TMT
}

Carl Nissly, Byoung-Joon Seo, Mitchell Troy, George Angeli, Myung Cho, et al.

Carl Nissly, Byoung-Joon Seo, Mitchell Troy, George Angeli, Myung Cho, Brent Ellerbroek, Piotr Piatrou, Lewis C. Roberts, J. Chris Shelton, Lianqi Wang, "High fidelity optical modeling for the TMT," Proc. SPIE 8336, Integrated Modeling of Complex Optomechanical Systems, 83360B (10 November 2011); doi: 10.1117/12.918708

SPIE Event: Integrated Modeling of Complex Optomechanical Systems, 2011, Kiruna, Sweden 


\title{
High Fidelity Optical Modeling for the TMT
}

\author{
Carl Nissly ${ }^{a, b}$, Byoung-Joon Seo ${ }^{a, b}$, Mitchell Troy ${ }^{a, b}$, George Angeli ${ }^{c}$, Myung Cho $^{d}$, \\ Brent Ellerbroek $^{c}$, Piotr Piatrou ${ }^{e}$, Lewis C. Roberts Jr. ${ }^{a, b}$, J. Chris Shelton ${ }^{a, b}$, Lianqi Wang ${ }^{c}$ \\ ${ }^{a}$ Jet Propulsion Laboratory, Pasadena, CA. 91109, USA \\ ${ }^{b}$ California Institute of Technology, CA. 91125, USA \\ ${ }^{c}$ Thirty Meter Telescope Observatory, CA. 91105, USA \\ ${ }^{d}$ National Optical Astronomy Observatory, AZ. 85719, USA \\ ${ }^{e}$ University of California, Irvine, CA. 92697, USA
}

\begin{abstract}
The Thirty Meter Telescope (TMT) is a Ritchey-Chritien optical telescope with a 30-meter diameter primary mirror made up of 492 hexagonal segments. Such a large and complex optical system requires detailed modeling of the optical performance during the design phase. An optical modeling computational framework has been developed to support activities related to wavefront \& image performance prediction. The model includes effects related to mirror shape sensing \& control, mirror alignment \& phasing, M1 segment control, low order wavefront correction, adaptive optics simulation for high order wavefront correction, and high contrast imaging. Here we give an overview of this optical simulation framework, the modeling tools and algorithms that are used, and a set of sample analyses. These tools have been used in many aspects of the system design process from mirror specification to instrument \& sensor design to algorithm development and beyond.
\end{abstract}

Keywords: Thirty Meter Telescope, Normalized Point Source Sensitivity, Optical Modeling, MACOS, APS, TMTracer, M1CS, OIWFS, NFIRAOS, MAOS, PFI

\section{INTRODUCTION}

\subsection{Optical Modeling Computational Framework}

The design of a telescope as large and complex as the Thirty Meter Telescope (TMT) requires significant engineering design and development prior to construction due to its significant increase in complexity and cost over existing observatories. This rise in complexity grows naturally with the size of the telescope aperture as requirements for thermal and structural stability become more strict and wavefront sensing and control of many more controllable degrees of freedom become more challenging. This design process requires an ability to evaluate the telescope's image performance for the broad range of requirements, system disturbances, and instrumentation necessary to achieve the science goals of the system. This is the motivation behind the development of the robust TMT optical performance simulation described here.

The TMT design team has developed a tool that can determine optical performance for a suite of disturbance inputs and parametric trade studies. This has been accomplished by distributing the work across each discipline; giving responsibility to each group to develop a standalone tool for simulation specific to their work. The individual tools are then connected together to operate within an end-to-end optical simulation. This choice in distributing the responsibility gives the advantages of allowing each group to use the programming language and modeling environment of their choice. Each developer can then simulate the performance of their system function independent of the entire group. The individual simulation routines are then combined in an overall performance tool that runs in the Matlab environment. A set of Matlab routines are used to manage the data interfaces between each element.

We refer to this end-to-end optical performance simulation tool for TMT as the Optical Modeling Computational Framework. The individual elements, data interfaces, and image metric products that make up the simulation framework are described in Figure 1. The model inputs from thermal and structural modeling as well

Further author information: (Send correspondence to Carl Nissly) E-mail: Carl.R.Nissly@jpl.nasa.gov

Integrated Modeling of Complex Optomechanical Systems, edited by Torben Andersen, Anita Enmark, Proc. of SPIE Vol. 8336, 83360B - () 2011 SPIE · CCC code: 0277-786X/11/\$18 · doi: 10.1117/12.918708 
as requirements such as mirror surface specifications are shown on the left and described in Section 1.2. These blocks each pass rigid body and mirror shape states to a Matlab Preprocessor, which handles the intermediate processing necessary to manage data interface issues.

The Matlab Preprocessor then interfaces with three additional telescope elements responsible for system calibration. First, the Mirror Shape Sensing and Control simulation measures the shape errors on each segment and calculates the correction imposed by the segment warping harnesses (WH). This simulation is described in further detail in Section 2.2. Second, the Alignment and Phasing System (APS) simulates the full optical sensing and calibration of the telescope aberrations and misalignments. Section 2.3 describes the capabilities of the APS simulation in detail. Third, the M1 Segment Control System (M1CS) simulation is responsible for maintenance of segment alignment with inputs from segment edge sensors, optical wavefront sensors, and calibration tables. This tool is explained in Section 2.4.

After state inputs are passed from each of the calibration, thermal, and structural simulations, The Matlab Preprocessor passes the telescope state in the form of rigid body and mirror shape perturbations to the Wavefront Calculation routine. This core simulation block is described in Section 2.1. The telescope Optical Path Difference (OPD) map is passed to a Matlab Postprocessor routine which then calculates the Point Spread Function (PSF) and image metrics such as the RMS wavefront error (RMS WFE), Normalized Point Source Sensitivity (PSSN), ${ }^{1-3}$ plate scale distortion, pupil shift, and pointing error.

For further instrument and performance simulation these image metrics along with the OPD map are passed to the simulation blocks shown on the right hand side of the Optical Modeling Framework diagram. First, the High Order Wavefront Correction simulation estimates the Adaptive Optics (AO) telescope performance. The tool used for this analysis has been developed in house by TMT and is described in Section 2.5. Second, the Low Order Wavefront Correction simulation models the telescope performance after slow time scale correction of lower spatial frequency errors such as thermal and gravitational misalignment. Section 2.6 describes this model. Third, the PSSN estimator is a systems engineering tool developed for building up the telescope error budgets. Individual error terms are run through the system to build look up tables that can analyze the telescope performance for varying combinations of error terms and environmental conditions. Finally, the High Contrast Imaging performance of the TMT Extreme AO (ExAO) instrument called the Planet Formation Imager (PFI) is described in Section 2.7. This simulation uses its own algorithm pipeline much like that of the Optical Modeling Framework described here to estimate the telescope performance in terms of the achievable contrast ratios when observing very faint objects close to stars.

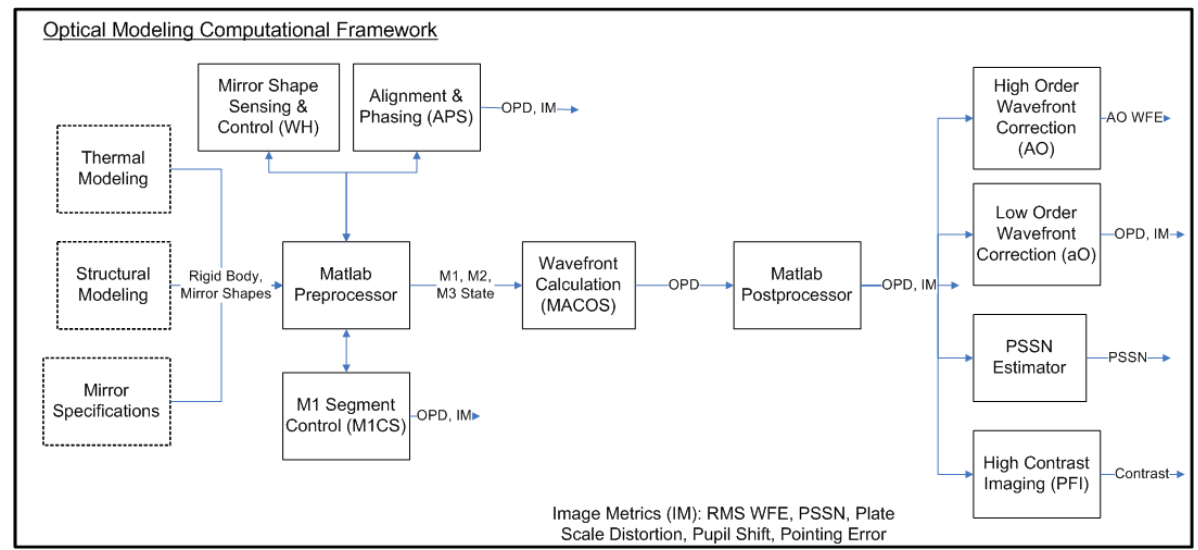

Figure 1: Optical Modeling Framework

\subsection{Model Inputs}

This Optical Modeling Framework is constructed to analyze a broad set of input error terms. These primarily come from three sources. First, the system requirements define the mirror specifications such as surface polishing, passive support performance, and installation tolerances. These terms are modeled directly as input to the framework by translating them into a set of statistics on rigid body positions and surface deformation grids. 
Other terms such as operational temperatures, wind loading, structural properties, and disturbance sources must first be modeled thermally and structurally. After these analyses are complete. a set of rigid body positions and surface deformation grid sensitivities can be computed for the various input parameters defined by the requirements. Some of these are static sensitivities such as mirror motion per degree temperature change, while others are time dependent and a time series of inputs is passed into the framework. Specific information for many of these model inputs relating to static terms, time dependent, thermal errors, and structural dynamics errors can be found in $\left[\right.$ Nissly-2008], ${ }^{4}\left[\right.$ Nissly-2010], ${ }^{5}\left[\right.$ Cho-2011], ${ }^{6}$ and $\left[\right.$ MacMynowski-2011] ${ }^{7}$ respectively.

\section{SIMULATION TOOLS AND ALGORITHMS}

\subsection{Wavefront Calculation}

At the heart of the Optical Modeling Framework is the Wavefront Calculation model. This simulation of the TMT wavefront is conducted using the JPL developed tool called Modeling and Analysis for Controlled Optical Systems (MACOS). MACOS has the ability to perform both ray-tracing and diffraction calculations. It has the advantage of efficient OPD calculation for highly segmented systems like TMT as shown in Figure 2. For our wavefront calculation, we utilize the ray-tracing capability inherent to MACOS and then interface this information to Matlab for all other diffraction and system state related computation. Once MACOS has calculated the telescope OPD map based upon a set of input parameters relating the rigid body positions and surface deformation states, our Matlab routines can calculate optical performance metrics. This includes the TMT OPD and PSF, RMS WFE, PSSN, plate scale distortion, pupil shift, and pointing error. This work has been reported in detail previously in [Nissly-2008]. ${ }^{4}$

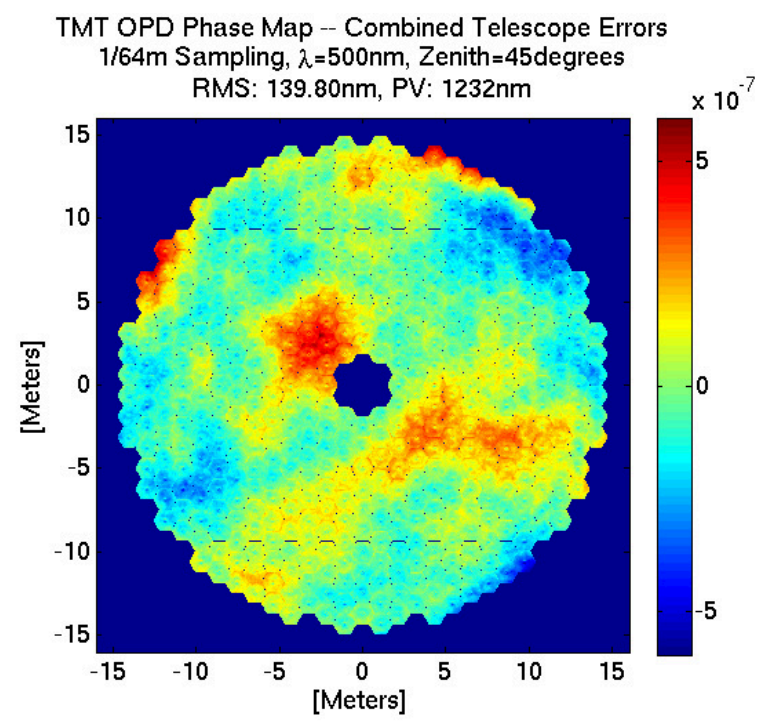

Figure 2: $\quad$ TMT OPD map for combined static telescope errors giving an RMS WFE of 133.103nm at Zenith angle $=45 \mathrm{deg}, \lambda=500 \mathrm{~nm}$.

\subsection{Mirror Shape Sensing and Control}

To simulate the active surface correction of the TMT primary mirror segments, the Mirror Shape Sensing and Control model is used. This simulation includes the data flow highlighted in Figure 3. First, the mirror specification defines the spatial frequency content and magnitude of the uncorrected segment shape errors. This information is then passed to the Matlab preprocessor routine where an uncorrected mirror segment shape is statistically generated. This surface is then passed to the Mirror Shape Sensing and Control model for correction. Within this Matlab routine, a Shack-Hartmann wavefront sensor (SH WFS) measures the initial segment shape error and a set of segment WH commands are calculated to minimize the residual surface error. Once this calculation is complete for the 492 segments that make up the TMT primary mirror, the segment deformations 
are passed through the Matlab preprocessor to the Wavefront Calculation model where the optical performance metrics are computed.

This model has variable parameters specific to both the wavefront sensing and control models. The simulation has led to a change in the baseline number of lenslet samples across each segments from 3 rings of hexagonal lenslets to 5 rings. The model has also been used to determine the optimum number of warping harness SVD modes to control by minimizing both the residual surface error and actuator stroke used to obtain the correction. Further detail for these analyses can be found in [Seo-2009]. ${ }^{8}$

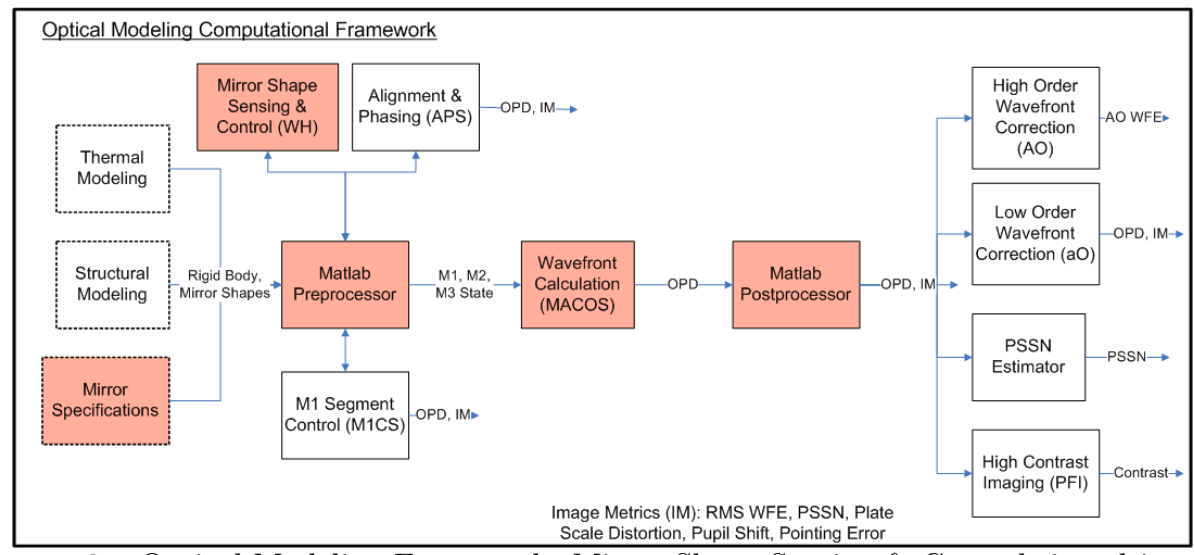

Figure 3: Optical Modeling Framework: Mirror Shape Sensing \& Control simualtion flow

\subsection{Alignment and Phasing System}

The Alignment and Phasing System is the TMT instrument that will be responsible for optical sensing of the telescope aberrations and misalignments and for generating the correction commands for the primary (M1), secondary (M2) and tertiary (M3) mirror control systems. ${ }^{9}$ The APS will also be used for creating look-up tables for deterministic telescope perturbations such as gravity for systematic error rejection between APS runs.

The core of the APS simulation software is a collection of data processing algorithms associated with the telescope alignment: tomographic and non-tomographic controllers for the TMT active optics correction and phasing. Development of these controllers required creating a software testbed infrastructure for performing simulations of the APS-TMT interaction. TMTracer, ${ }^{10}$ a software suite tailored to simulation of the optical alignment of extremely large segmented telescopes, is a result of our efforts taken in this direction. The purpose of TMTracer is first to provide simulation tools to facilitate the APS development process. Second, since the APS control strategies are model-based, to eventually transfer the models and control algorithms developed with the aid of TMTracer to serve the physical instrument.

TMTracer is developed in house for TMT using Fortran running on Linux. The code has extensive capability to simulate the end-to-end telescope commissioning process for TMT. Major features and abilities of the code are as follows:

- Embedded models for segmented telescope optical systems with arbitrary perturbations, Shack-Hartmann and segment step wavefront sensors for the APS instrument and also low-order on-instrument wavefront sensors.

- Visualization of system geometrical state, sensor measurements and mechanism commands; state reports and telemetry records for all parts of the system.

- Sequential ray tracing engine with productivity of $100 \mathrm{~K}$ rays/sec traced through an arbitrarily perturbed giant segmented 3-mirror telescope.

- Optical performance metrics: ray diagrams on a surface, in nominal and optimal focus, OPD, PSF, OTF, EE, PSSN, focal surface shape, position, image scale error, and distortion. 
- Collection of least squares control algorithms including tomography; an internal calibration system for numerical computation of observability matrices; LibCell library for efficient handling of large complexity sparse structured data; observability analysis facilities.

- End-to-end Monte-Carlo simulator with data visualization and recording system.

The TMTracer software currently functions independent of the Optical Modeling Framework since it has the capability to simulate the entire telescope alignment and phasing process from input errors to wavefront calculation to correction commands. Therefore the current implementation of the Optical Modeling Framework includes approximations of the TMTracer output in the form of rigid body commands. The shape correction is currently implemented using the Mirror Shape Sensing and Control model described in Section 2.2. Integrating the input and output of the TMTracer software remains a goal for added maturity to the Optical Modeling Framework since the APS system performance is key to other telescope elements such as the M1 control system as an example.

\subsection{M1 Control System}

The TMT primary mirror control system actively controls 1476 segment actuators, with real-time inputs from 2772 edge sensors on the segments and optical feedback from either the On-Instrument Wavefront Sensor (OIWFS) or the NFIRAOS adaptive optics system. The M1CS control system is itself calibrated using accumulated data from several APS runs. The M1CS Simulation was created to study the complex interaction of all of these elements. $^{7}$

The M1CS Simulation is a time-domain iterative model, capable of modeling effects such as sensor and actuator nonlinearity and saturation. It takes as inputs the TMT Segmentation Database, which specifies the 492 irregular hexagonal segments that together make up the f/1 primary mirror, and a master file with the results of FEA modeling of gravity deformations. It applies segment and sensor installation errors, simulates the APS runs, and the action of the segment warping harnesses. With the simulated loop closed, the 3D positions and orientations of all M1CS elements are tracked, with changes in gravity and temperature allowed during the closed-loop operation. Optical input from an OIWFS or AO is modeled as a series of Zernike coefficients, with a control loop added to drive each coefficient to zero. A detailed sensor model includes the effects of segment in-plane motions, such as changes in the gaps between segments.

A second major piece of the simulation is to model M1CS calibration. Calibration compensates for the change in edge sensor gains and offsets due to unavoidable changes in the gaps between segments. The calibration code collects data from several simulated APS runs at different zenith angles and temperatures, and computes coefficients of a fit function, or correction formula for the sensor offsets. The calibration modeling software has allowed the comparison of several candidate fit functions, and the evaluation of the performance of the calibration concept. These capabilities are essential to TMT since the intent of the simulation is that it will evolve into actual run-time software for TMT.

The simulation is written in Matlab. Its connection with the Optical Modeling Framework is that the MACOS Wavefront Calculation and PSSN Estimator are used to derive the PSSN metrics for calibration errors. The M1CS Simulation has the inherent capability to generate surface maps showing the state of the primary mirror. The simulation can also pass its rigid body state to the Matlab Preprocessor for telescope wavefront calculation and PSSN image performance calculation.

\subsection{High Order Wavefront Correction}

The Multi-Threaded Adaptive Optics Simulator (MAOS) is the AO simulation developed by TMT. ${ }^{11,12}$ It is a completely new implementation of the MCAO simulator LAOS, which was written in MATLAB language and used by TMT previously. The motivation to develop this software is to create a multi-conjugate adaptive optics simulator that runs fast, consumes less memory, and does the job without MATLAB, which is proprietary and has a large memory footprint. MAOS is written with a function oriented design and is completely configurable through configuration files. The code tries its best to check the configuration for any apparent errors or conflicts. The configuration files are easily readable and maintained. The final development goal is to make an efficient, 
easy to use, general purpose adaptive optics simulator to help the development of adaptive optics systems, particularly advanced, very high order systems for TMT and other ELTs.

The typical usage of this software is to assess the performance of an AO system, which can be either single conjugate, multi conjugate, laser tomographic, ground layer, or multi-object AO system. It can compute the open loop and closed loop wavefront error for multiple science targets and their average with low order Zernike modes removed (optional). It can also compute open and closed loop PSFs of the science targets. This allows the MAOS tool to calculate AO system performance for a broad range of specified input and operational conditions.

The specific implementation of the MAOS software for TMT is used in connection with the Narrow Field InfraRed Adaptive Optics System (NFIRAOS). NFIRAOS is the early-light AO system for $\mathrm{TMT}^{13,14}$ and is a multi-conjugate $\mathrm{AO}$ system (MCAO) that will utilize 6 laser guide-stars and two deformable mirrors to provide diffraction-limited atmospheric turbulence compensation at near IR wavelengths over a 10"-30" FoV. A fully functional simulation of the NFIRAOS capabilities and performance is implemented using MAOS. This simulation has been used for the extensive engineering tasks of the design and system engineering for the system.

This software is written in the C language (revision 99), with external dependent libraries of FFTW version 3 and blas/lapack. The code contains a local copy of the package Arpack and Cholmod. An optimized blas library such ATLAS, GOTOBLAS, or Intel MKL is necessary to get good performance for Cholesky decompositions of the tomography and/or fitting matrix.

\subsection{Low Order Wavefront Correction}

During an observational night, a large telescope such as TMT experiences system misalignment due to thermal and gravitational effects. The Low Order Wavefront Correction model simulates the correction of these errors based on low order SH WFS measurements mounted on a seeing limited instrument. We refer to this sensor as an On-Instrument Wavefront Sensor. This OIWFS simulation currently models the time dependent mirror misalignments estimated by the TMT thermal model. ${ }^{6}$

The OIWFS simulation is developed using Matlab. A 3 ring SH WFS is simulated across the TMT pupil to measure up to third order errors. A Type- 1 servo controller with velocity control is simulated to send commands to the M2 rigid body translation to correct for the telescope misalignments. Atmospheric noise is included using time averaged values. This gives the model the capability of varying the OIWFS design, controllable degree of freedom parameters, command update rate, integration time, as well as the number and position of OIWFSs used.

In the context of the Optical Modeling Framework, this simulation effectively utilizes a feedback loop through the framework. Meaning that the Low Order Wavefront Correction block senses slope measurements and calculates control commands from the wavefront passed from the Matlab Postprocessor, the Matlab Preprocessor adds these commands to the time dependent disturbance and a new telescope wavefront is computed. The telescope image metrics are computed for each time step as measured across the telescope field. Further details regarding this simulation can be found in [Nissly-2010]. ${ }^{5}$

\subsection{High Contrast Imaging}

Three techniques have been implemented to study the performance for the TMT Planet Formation Imager (PFI) ${ }^{15}$ Analytical error models provide useful first order estimates of system performance, but ultimately numerical simulations are needed. Thus, specific numerical simulations were used to model distinct aspects of the system that could not be modeled analytically or to verify the analytical calculations. We also developed an end-to-end numerical simulation to capture as much of the system behavior as possible and to validate the analytic and independent numerical simulations.

Different subsystems were being designed by separate institutions and required varying code bases to analyze their performance. Thus an elegant solution was to write independent codes to analyze the various sub-systems and then to link them together to form a full simulation. Much like the overall Optical Modeling Framework, the PFI simulation distributes computation across various models and unifies the process in an ensemble of routines. Each algorithm writes out files (in a specified format) that are then read in by the next algorithm in the chain. This allows individuals to work on, optimize, and analyze the individual portions (algorithms) of 
the simulations. Figure 4 shows the algorithm pipeline that we developed. The atmosphere algorithm calculates and writes to files the complex pupil at the telescope aperture from the atmosphere as a function of time. The telescope algorithm can approximate a complex pupil that represents the telescope introduced phase and amplitude errors independent of the Optical Modeling Framework. Alternatively, the output of the Matlab Postprocessor framework block can replace this telescope model to use the errors implemented through the MACOS simulations. The atmosphere and telescope pupils are fed into a simulation of the pre-DSS (Diffraction Suppression System) AO system, which writes out the corrected complex field as a function of time. The DSS algorithm can simulate a number of different methods for suppressing the starlight, although in this study we concentrated on the nuller. The post-DSS WFS senses the wavefront and controls a DM in the DSS to correct the wavefront. The output of this algorithm is both the corrected complex pupil and an estimate of the complex pupil as measured by the WFS. The corrected complex pupil is read into an algorithm to simulate the science camera that produces co-added PSFs. Finally, a post-processed image is created using the estimated PSF from the WFS and the science focal plane.

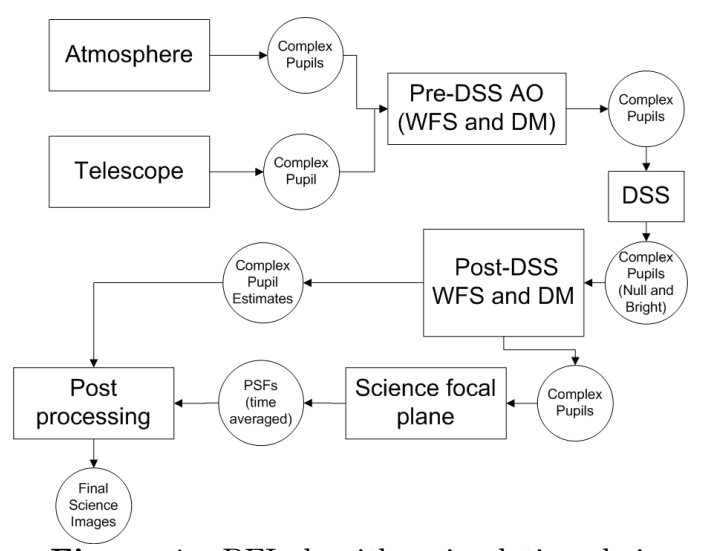

Figure 4: PFI algorithm simulation chain

\section{SAMPLE ANALYSES}

The tools described in Section 2 provide a powerful set of models to simulate the optical performance for the telescope under a wide variety of input parameters. Trade studies can be conducted in areas specific to each discipline such as thermal and structural modeling in terms of optical performance, mirror surface polishing specification, APS instrument design and algorithm trades, control law parametric trades, and PSF generation for instrument design studies and performance analyses. The end-to-end Optical Modeling Framework not only allows for these discipline level optimizations, but also for system engineering error budget development and validation by connecting the individual blocks to function within the framework. Section 3 describes a sample set of analyses that utilize the capabilities of the individual blocks functioning corporately to provide significant added benefit.

\subsection{Alignment and Phasing System Performance}

A typical use-case for the TMTracer software that simulates the APS systems for TMT is to input an error in the system and demonstrate the correction of that error by the controllable degrees of freedom. Figure 5 shows an example of this process by introducing a passive support error on M2. TMTracer then simulates the wavefront sensing of the perturbed telescope and calculates the necessary commands to optimize the wavefront. As a performance trade study, here the M1 segments are adjusted in rigid body motion and shape adjustment to correct for this input error on the M2 surface. This sample analysis demonstrates a simple use of TMTracer, while a more complex example simulation would include the full measurement and alignment of the telescope using the tomographic controllers currently in development. Fully developing the interface between the TMTracer simulation and the Optical Modeling Framework remains a necessary next step for the development of the framework. 

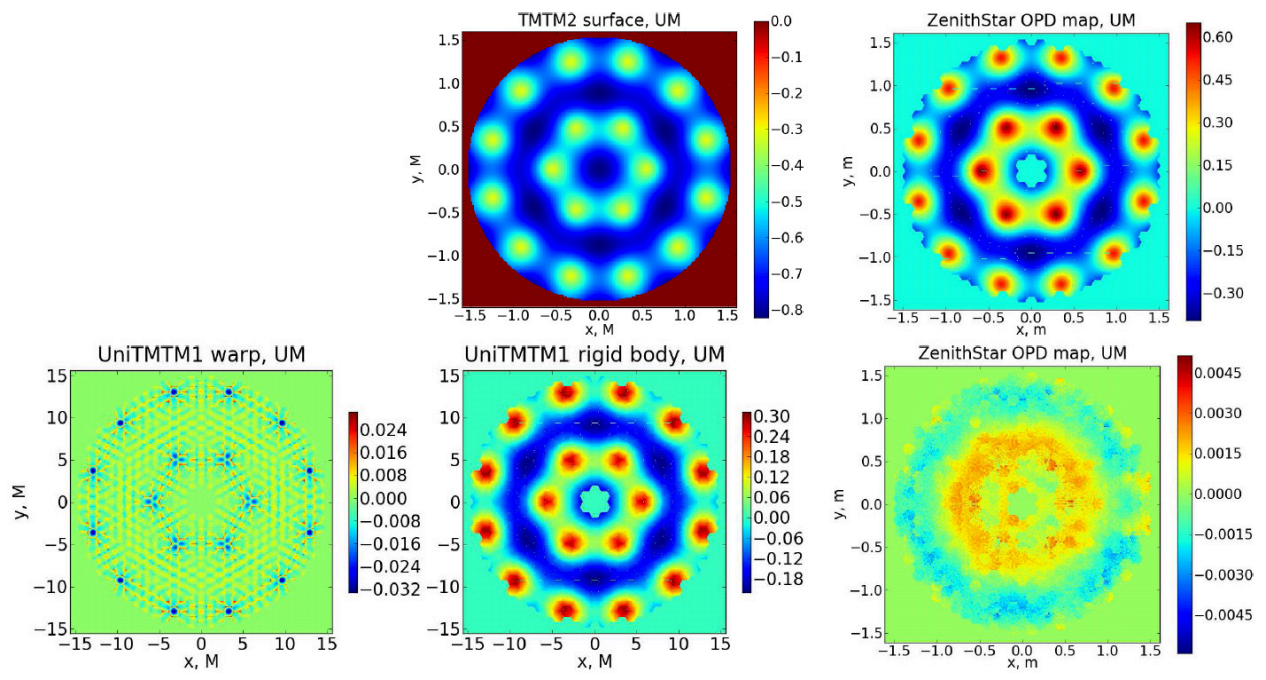

Figure 5: Upper row: M2 support print-through map (left) and OPD map of the corresponding wavefront error (right). Lower row from left to right: M1 segment shape corrections, M1 rigid body corrections, and net corrected TMT OPD maps. (Note change in scale for each figure.)

\subsection{M1 Control System Performance}

Components of the M1CS Simulation have been used as building blocks for other simulations and studies. The simulation has produced, for example, studies of the effects of sensor installation errors and of different edge sensor types, and studies of the impact of the system interaction matrix changing dynamically due to segment in-plane motions.

Numerous parameters can be varied and the appropriate values are set by parameter files. These parameters include the edge sensor type, installation errors for segments and sensors, sensor noise level, the number of Zernike modes to be sensed and removed externally by the adaptive optics system or instrument wavefront sensor, and a series of zenith angle/temperature pairs at which observations are simulated.

The parameter files also control the number and type of outputs. One type of output is a pupil graph, as shown in Figure 6. The left figure shows the M1 surface error after APS rigid body alignment but before warping, with typical segment and sensor installation errors. The right figure shows the same figure after segment warping is applied and the M1CS loop closed. Notice the 100x change of scale. This type of surface error analysis has led to the implementation of the interface to the Optical Modeling Framework. By passing states to the Matlab Preprocessor for Wavefront Calculation, performance can also be evaluated in terms of wavefront error and PSSN image performance.

\subsection{Adaptive Optics Performance}

The high order wavefront correction using TMT's MAOS tool has been used to simulate the NFIRAOS correction of static optical errors described in Section 1.2. These NFIRAOS simulations use the wavefront sensing parameters described in Section 2.5 and in greater detail by Gilles, Wang, and Ellerbroek. ${ }^{11,12}$ These LGS MCAO simulations utilize a conjugate gradient control algorithm, which can take many iterations to converge when initializing the simulation from scratch. This led to a simplified simulation case used for the context of the Optical Modeling Framework. Here natural guide star (NGS) and single conjugate AO system (SCAO) simulation settings provide an accurate approximation of the more complex wavefront controller for only a few simulated control iterations. Table 1 shows results for simulating the correction of individual static error terms in this way with no atmospheric or wavefront sensor noise. For the case where all static error terms relating to M1, M2, and M3 combined are simulated through the full simulation framework, the open loop wavefront error performance was approximately $140 \mathrm{~nm}$ and is corrected to approximately $41 \mathrm{~nm}$. Figure 7 shows the open and closed loop wavefront where the low and mid-spatial frequency terms have been corrected and segment level high frequency wavefront errors remain. 


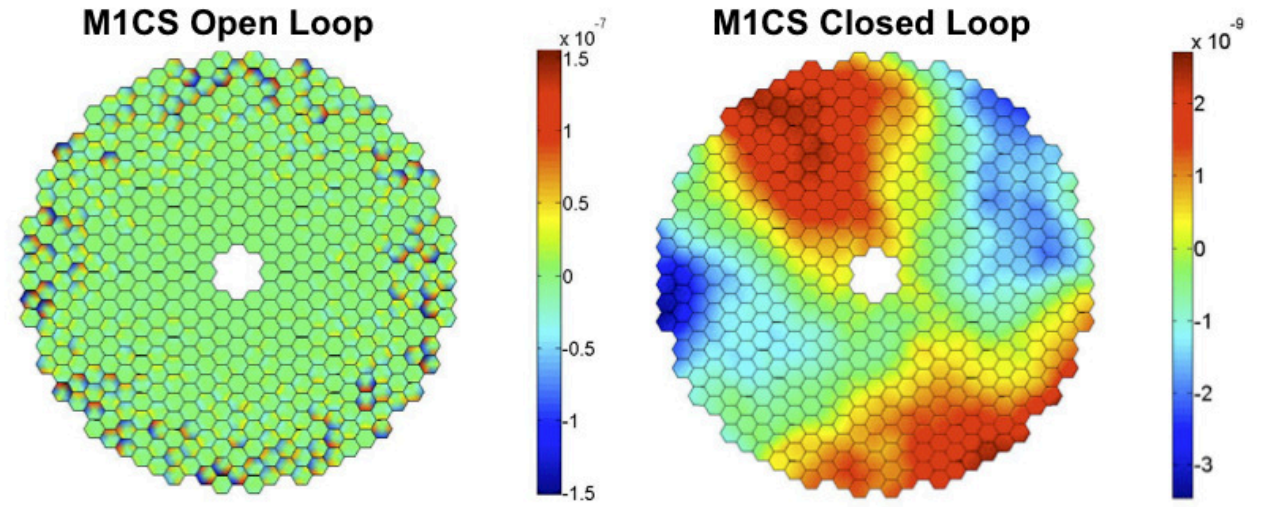

Figure 6: Typical run-time primary mirror surface error in meters. (Left) After APS simulation, but before warping. (Right) After APS and warping have been applied, and the M1CS loop is closed. Note the 100x scale reduction after M1CS surface correction.
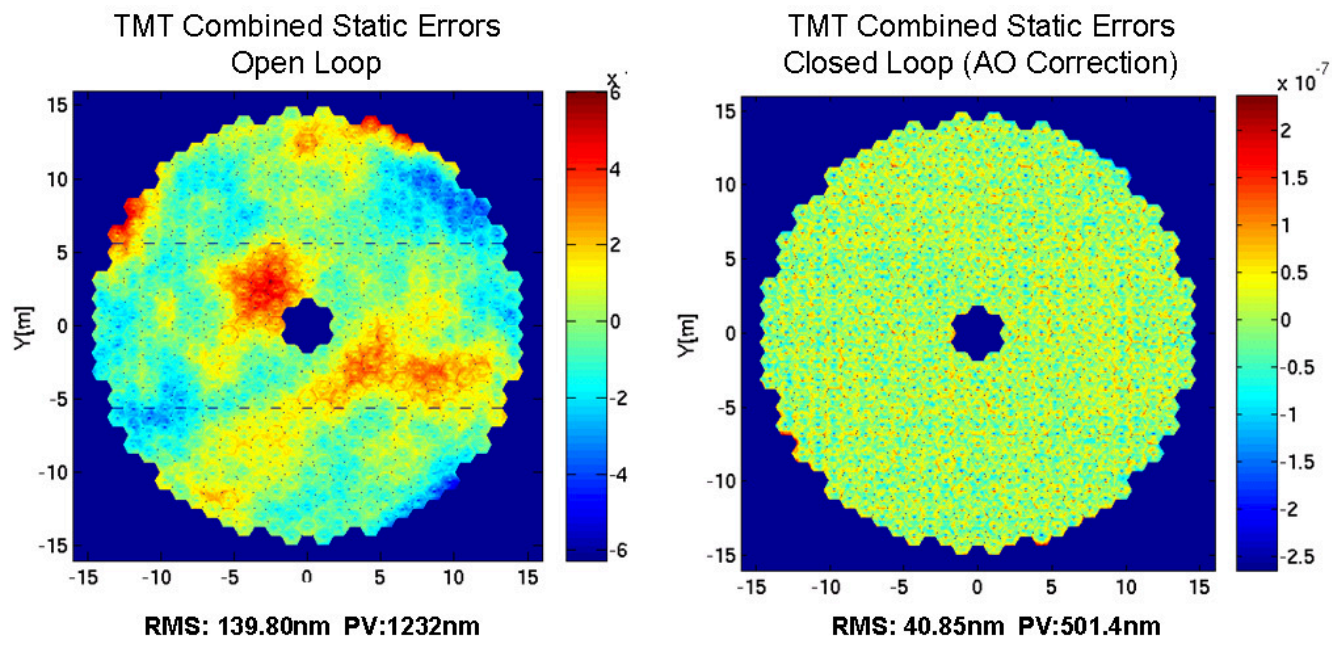

Figure 7: M1, M2, M3 combined static telescope errors open loop (left) and closed loop (right).

\section{SUMMARY}

The Optical Modeling Framework developed for TMT optical performance analysis has grown to become a powerful tool to assess a broad range of system disturbances and errors. Individual tools have been developed by separate disciplines to both distribute the work and provide a convenient method of simulating performance specific to each subsystem. These simulation tools include analysis capabilities specific to APS, M1CS, AO, ExAO, and overall system performance for systems engineering purposes. A set of interfaces between each element provide the capability to analyze a broad set of simulation parameters in terms of optical image metrics.

Figure 8 shows the level of maturity for each of the individual elements. Elements fully implemented into the framework include Mirror Shape Sensing and Control, Wavefront Calculation, High and Low Order Wavefront Correction, and the PSSN Estimator routines. The M1 Segment Control element has been partially implemented into the framework, meaning that the simulation can currently setup its own runtime parameters and evaluate performance through the framework in terms of a reduced set of image metrics. The Alignment and Phasing System element currently operates independent of the framework. This means that the simulation has the capability to independently simulate the telescope alignment process based upon its own set of inputs and tools. As mentioned previously, it is an essential next step to fully integrate the APS simulation into the framework. This will be achieved by developing an interface to the Matlab Preprocessor. Last, the High Contrast Imaging element currently functions independent of the framework for the most part. This is due to timing of the framework development. A significant amount of new inputs and optical modeling work has taken place since 
Table 1: NFIRAOS Corrected Wavefront Error

\begin{tabular}{|c|c|c|c|}
\hline \multirow{2}{*}{\multicolumn{2}{|c|}{$\mathrm{ZA}=60 \mathrm{deg}, \Delta \mathrm{T}=4$ degrees, $\mathrm{SCAO}, \mathrm{NGS}$}} & Open Loop & Closed Loop, Iter $=20$ \\
\hline & & RMS WFE [nm] & RMS WFE [nm] \\
\hline \multirow{9}{*}{ M1 } & Segment Passive Support - Axial & 9.78 & 10.34 \\
\hline & Segment Passive Support - Lateral & 21.18 & 28.86 \\
\hline & Gravity Segment Clocking \& Decenter & 5.05 & 2.85 \\
\hline & Thermal Segment Clocking & 2.43 & 1.58 \\
\hline & Thermal Segment Distortion & 19.74 & 7.81 \\
\hline & APS Random Segment Phasing & 13.69 & 5.79 \\
\hline & APS Correlated Segment Position & 142.14 & 8.38 \\
\hline & $\begin{array}{l}\text { Segment Figuring Error with } \\
\text { Warping Harness Correction }\end{array}$ & 24.54 & 23.75 \\
\hline & Combined M1 Errors & 147.35 & 41.04 \\
\hline \multirow{5}{*}{ M2 } & Segment Passive Support - Axial & 9.10 & 0.55 \\
\hline & Segment Passive Support - Lateral & 4.56 & 0.50 \\
\hline & APS Position Error & 90.18 & 5.44 \\
\hline & Figuring Error & 116.13 & 8.07 \\
\hline & Combined M2 Errors & 140.79 & 9.68 \\
\hline \multirow{5}{*}{ M3 } & Segment Passive Support - Axial & 9.40 & 0.45 \\
\hline & Segment Passive Support - Lateral-y & 0.54 & 0.03 \\
\hline & Segment Passive Support - Lateral-x & 0.71 & 0.03 \\
\hline & Figuring Error & 140.50 & 6.71 \\
\hline & Combined M3 Errors & 138.88 & 6.89 \\
\hline All & Combined M1, M2, M3 Errors & 139.80 & 40.85 \\
\hline
\end{tabular}

the last PFI simulations have been run. An update to the simulation interface and results would be a valuable assessment of the current prediction of telescope performance. Clear next steps for the development of the Optical Modeling Framework described here include updating the interfaces to the APS and PFI simulations and to continue utilizing the framework to determine system requirements and specifications for this powerful tool.

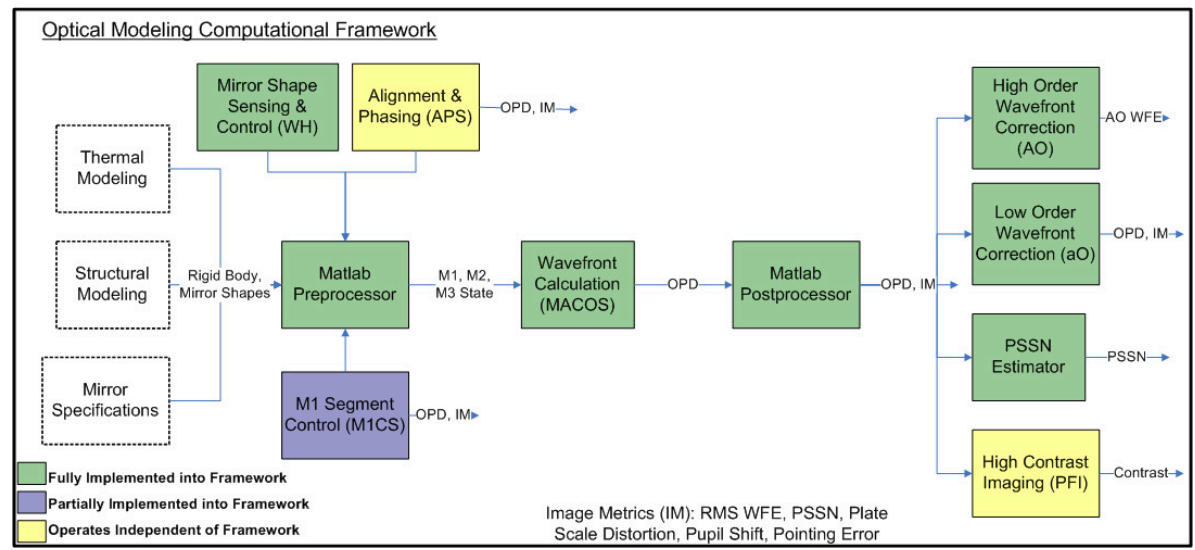

Figure 8: Optical Modeling Framework: Maturity

\section{ACKNOWLEDGMENTS}

This research was carried out in part at the Jet Propulsion Laboratory, California Institute of Technology, and was sponsored by the California Institute of Technology and the National Aeronautics and Space Administration. The authors gratefully acknowledge the support of the TMT partner institutions. They are the Association of 
Canadian Universities for Research in Astronomy (ACURA), the California Institute of Technology and the University of California. This work was supported as well by the Gordon and Betty Moore Foundation, the Canada Foundation for Innovation, the Ontario Ministry of Research and Innovation, the National Research Council of Canada, the Natural Sciences and Engineering Research Council of Canada, the British Columbia Knowledge Development Fund, the Association of Universities for Research in Astronomy (AURA) and the U.S. National Science Foundation.

\section{REFERENCES}

1. B.-J. Seo, C. Nissly, G. Angeli, B. Ellerbroek, J. Nelson, N. Sigrist, and M. Troy, "Analysis of Normalized Point Source Sensitivity as a performance metric for the Thirty Meter Telescope," Proc. SPIE 7017, p. 70170T, June 2008.

2. B.-J. Seo, C. Nissly, G. Angeli, B. Ellerbroek, J. Nelson, N. Sigrist, and M. Troy, "analysis of Normalized Point Source Sensitivity as performance metric for large telescopes," Applied Optics 48, pp. 5997-6007, 2009.

3. B. Seo, C. Nissly, M. Troy, and G. Angeli, "Normalized point source sensitivity for off-axis optical performance evaluation of the Thirty Meter Telescope," Proc. SPIE 7738-16, June 2010.

4. C. Nissly, B. Seo, M. Troy, G. Angeli, J. Angione, I. Crossfield, B. Ellerbroek, L. Gilles, N. Sigrist, and L. Wang, "High-resolution optical modeling of the Thirty Meter Telescope for systematic performance trades," Proc. SPIE 7017, p. 70170U, June 2008.

5. C. Nissly, B. Seo, M. Troy, G. Angeli, M. Cho, C. Shelton, N. Sigrist, and M. Sirota, "Investigation of Thirty Meter Telescope wavefront maintenance using low order Shack-Hartmann wavefront sensors to correct for thermally induced misalignment," Proc. SPIE 7738-17, June 2010.

6. M. Cho, A. Corredor, K. Vogiatzis, and G. Angeli, "Thermal modeling of the TMT telescope," Proc. SPIE Integrated Modeling of Complex Optomechanical Systems Symposium, Aug 2011.

7. D. G. MacMynowski, P. Thompson, J. C. Shelton, L. C. Roberts, M. M. Colavita, and M. J. Sirota, "Control system modeling for the Thirty Meter Telescope primary mirror," Proc. SPIE Integrated Modeling of Complex Optomechanical Systems Symposium, Aug 2011.

8. B. Seo, C. Nissly, G. Angeli, D. Macmynowski, N. Sigrist, M. Troy, and E. Williams, "Investigation of primary mirror segment's residual errors for the Thirty Meter Telescope," Proc. SPIE 7427, Aug. 2009.

9. M. Troy, G. Chanan, S. Michaels, R. Bartos, G. Bothwell, R. Hein, M. Radin, J. Roberts, J. M. Rodgers, L. Scherr, B.-J. Seo, and D. Zimmerman, "A conceptual design for the Thirty Meter Telescope Alignment and Phasing System," Proc. SPIE, June 2008.

10. P.Piatrou and G. Chanan, "The TMTracer: a modeling tool for the TMT Alignment and Phasing System," Proc. SPIE 8149-3, Aug 2011.

11. L. Gilles, L. Wang, and B. Ellerbroek, "Modeling update for the Thirty Meter Telescope laser guide star dual-conjugate adaptive optics system," Proc. SPIE, June 2010.

12. L. Wang, "MAOS documentation," (http://lianqiw.github.com/maos/), 2011.

13. B. Ellerbroek, S. Adkins, D. Andersen, J. Atwood, C. Boyer, P. Byrnes, R. Conan, L. Gilles, G. Herriot, P. Hickson, E. Hileman, D. Joyce, B. Leckie, M. Liang, T. Pfrommer, J.-C. Sinquin, J.-P. Véran, L. Wang, and P. Welle, "Progress towards developing the TMT adaptive optical systems and their components," Proc. SPIE, June 2008.

14. G. Herriot, D. Andersen, J. Atwood, C. Boyer, P. Byrnes, B. Ellerbroek, L. Gilles, P. Hickson, J.-P. V. B. Leckie, L. Wang, and P. Welle, "NFIRAOS - TMT's initial adaptive optics system," Proc. SPIE, June 2008.

15. B. Macintosh, M. Troy, R. Doyon, J. Graham, K. Baker, B. Bauman, C. Marois, D. Palmer, D. Phillion, L. Poyneer, I. Crossfield, P. Dumont, B. M. Levine, M. Shao, G. Serabyn, C. Shelton, G. Vasisht, J. K. Wallace, J.-F. Lavigne, P. Valee, N. Rowlands, K. Tam, and D. Hackett, "Extreme adaptive optics for the Thirty Meter Telescope," Advances in Adaptive Optics II. Edited by Ellerbroek 6272, p. 20, July 2006. 